\title{
Web Composition with WCAG in mind
}

\author{
Vicente Luque Centeno, Carlos Delgado \\ Kloos \\ Carlos III University of Madrid \\ \{vlc,cdk\}@it.uc3m.es
}

\author{
Martin Gaedke, Martin Nussbaumer \\ University of Karlsruhe \\ \{gaedke,nussbaumer\}@tm.uni- \\ karlsruhe.de
}

\begin{abstract}
Accessibility should be a part of the Web design process instead of being a post-design repair process. Thus, it should be more integrated within the internal authoring tools' mechanism of generating new accessible Web contents. Web pages are usually composed of small pieces of HTML code which, dynamically nested and combined, generate full Web pages. This Web composition, specially when creating Web pages from data extracted from heterogeneous or external sources, should have accessibility into account in order to guarantee that the final page being constructed is accessible. This paper presents the set of rules that, in a Web composition process, a design tool must follow in order to create accessible Web pages. These rules are formalized with W3C standards like XPath and XQuery expressions. We also present WSLS as an accessibility enabled authoring tool that makes this task feasible, and focus in how this tool incorporates accessibility into the process of generating new Web contents.
\end{abstract}

\section{Keywords}

WAI; WCAG; Web composition

\section{Introduction}

Web browsers have evolved since their inception at the beginning of the Web. We can find plenty of them in very different and heterogeneous devices. Wireless devices like PDA, palm-tops or mobile phones can be connected to Internet, as well as other new devices like digital TV's, or vehicle's assistants. However, many difficulties (namely barriers) arise when these devices are used for surfing legacy Web sites, i.e., Web sites without accessibility features in their designer's mind.

Disabled people also experiment similar problems when using specific platforms adapted to their special needs, like Braille displays, voice browsers or screen magnifiers to Web sites having these accessibility barriers. As a result, ac-

Permission to make digital or hard copies of all or part of this work for personal or classroom use is granted without fee provided that copies are not made or distributed for profit or commercial advantage and that copies bear this notice and the full citation on the first page. To copy otherwise, to republish, to post on servers or to redistribute to lists, requires prior specific permission and/or a fee.

W4A at WWW2005 10th May 2005, Chiba, Japan

Copyright 2005 ACM 1-59593-036-1/05/05 ...\$5.00. cessibility has become a very important feature, and W3C defined WCAG $[1,2]$ as a set of guidelines that Web pages should follow in order to be considered accessible. The ability for a Web site to be surfed by any device has become a major target for Web designers.

However, most authoring tools still lack from features for generating accessible and device-independent markup. At this point, accessibility is usually treated as a post-design repair process, most of the times guided by an accessibility evaluator tool, like Bobby [9], Tawdis [10] or Torquemada [11] that spots accessibility barriers that specialized persons have to supervise with no little effort.

Removing accessibility barriers for already made Web sites involves a huge amount of human effort because a lot of human supervision is required for these evaluation tools. This post-design repair process is much less effective than having accessibility features in mind during the design process. Since authoring tools should produce high quality markup for new Web contents, we believe that most of accessibility benefits can be achieved if authoring tools implement accessible-aware creation process mechanisms that guarantee that their generated Web content will be accessible.

Web composition [12] has emerged as a consistent, flexible and dynamic way of generating Web contents by combining reusable pieces of HTML code into other reusable pieces that, guided by a composition process, will be used to create whole Web pages and whole Web sites. This is the approach of several authoring tools like WSLS [14], that extracts dynamic contents from heterogeneous sources and automatically generates new updated Web contents without the need of a person to guide the process in running time. For these tools, it would be highly desirable to guarantee that final Web contents will be accessible, provided that smaller pieces of reusable HTML code to be used in Web composition are already accessible. In other words, provided that a Web Composition based authoring tool manages small pieces of accessible HTML code (both static templates or dynamically generated) we want to guarantee that the Web composition process will always yield an accessible Web page (or site). So, we must make sure that no kind of new accessibility barriers can be introduced by the Web composition process itself. This paper is focused on formalizing the conditions to be met so that accessible chunks of Web pages can be safely compounded into a page that also results accessible from a WCAG point of view. As we will see, non accessible results can be obtained from accessible pieces, unless some rules are followed. This paper provides a formalized set of such rules. 


\section{WCAG compliant composition}

WCAG defines three accessibility levels, being A, AA and AAA. In order to declare some common names in our rules, we establish the following definitions.

1. Given $\$ \mathrm{~S} 1$ and $\$ \mathrm{~S} 2$ compoundable pieces of HTML markup. ${ }^{1}$

2. Given wailevel $(\$ S)=\{$ No|A $|\mathrm{AA}| \mathrm{AAA}\}$ (an ordered set, so that $\mathrm{No}<\mathrm{A}<\mathrm{AA}<\mathrm{AAA}) .^{2}$

3. Provided that snippets $\$ S 1$ and $\$ S 2$ are WCAG compliant, i.e., wailevel $(\$ \mathrm{~S} 1) \in\{\mathrm{A}|\mathrm{AA}| \mathrm{AAA}\} \wedge$ wailevel $(\$ \mathrm{~S} 2) \in\{\mathrm{A}|\mathrm{AA}| \mathrm{AAA}\} .^{3}$

4. Provided that snippet $\$ S 2$ will be nested inside snippet $\$ \mathrm{~S} 1$ and that $\operatorname{pos}(\$ \mathrm{~S} 1, \$$ position $)$ is the insertion point for $\$ \mathrm{~S} 2$ inside $\$ \mathrm{~S} 1$, as depicted in figure 1 . $^{4}$

5. Assuming bottom-up document construction, i.e., $\$ \mathrm{~S} 2$ has no position where other HTML reusable pieces might be inserted. ${ }^{5}$

6. Provided that comp $(\$ \mathrm{~S} 1, \$ \mathrm{~S} 2, \$$ position $)$ is the composition of $\$ \mathrm{~S} 2$ inserted into pos $(\$ \mathrm{~S} 1, \$$ position $)$, i.e., $\$ \mathrm{~S} 2$ nested somewhere in $\$ \mathrm{~S} 1$.



Figure 1: Web Composition of HTML snippets

The purpose of this section is to declare which conditions should be met so that wailevel $(\operatorname{comp}(\$ \mathrm{~S} 1, \$ \mathrm{~S} 2, \$$ position $)) \geq \mathrm{MIN}($ wailevel $(\$ \mathrm{~S} 1)$, wailevel $(\$ S 2)){ }^{6}$

\footnotetext{
${ }^{1}$ Also called HTML snippets.

${ }^{2}$ The accessibility level defined by WCAG from W3C for HTML snippet $\$ \mathrm{~S}$.

${ }^{3}$ This is required to apply induction. If all basic snippets are accessible and the composition process guarantees that, provided accessible snippets, the result is also accessible, then all composed contents will always be accessible (by induction).

${ }^{4}$ This insertion point can also be considered, for simplicity, as a virtual empty element within $\$ S 1$ that will be substituted by $\$ S 2$. This latter approach is applyable for Web composition based on technologies like, for example, JSP or ASP, because these formats have special tags whose purpose is to be replaced by some other contents when generating new Web pages.

${ }^{5}$ In other words, $\$ \mathrm{~S} 2$ has no free insertion point, while $\$ \mathrm{~S} 1$, at least has an insertion point for $\$ \mathrm{~S} 2$.

${ }^{6}$ We are not really interested on upgrading our pieces' accessibility level (though this could happen if both $\$ \mathrm{~S} 1$ nd $\$ \mathrm{~S} 2$ provide complimentary accessibility markup). Our focus resides on not degrading our pieces' accessibility level because of an unfortunate composition.
}

\section{Process-aware checkpoints}

This section covers all WCAG checkpoints that should be reviewed before allowing Web composition to produce results. This is because, even provided that $\$ \mathrm{~S} 1$ and $\$ \mathrm{~S} 2$ comply with them, comp $(\$ \mathrm{~S} 1, \$ \mathrm{~S} 2, \$$ position) might not comply if some conditions are not followed. Thus, this section covers all these extra conditions to be met in order to guarantee accessibility, provided $\$ \mathrm{~S} 1$ and $\$ \mathrm{~S} 2$ are accessible. If these conditions are not met, accessibility barriers could be incidentally introduced.

The way these conditions will be guaranteed by authoring tools can be rather different from one tool to another and is an issue of these tools. Some tools might stop the composition process with an error or exception if these required process-aware checkpoints are detected as not followed. Some tools might present a form so that the author indicates some extra required information or allow the operation under his responsibility. Some tools might automatically and transparently add new markup or contents in order to eliminate a possible barrier, ...

\section{Rule 1.4: Global synchronization of timed events}

Provided that both $\$ \mathrm{~S} 1$ and $\$ \mathrm{~S} 2$ have all their timed events (if any) internally synchronized (intra-synchronization), comp (\$S1,\$S2,\$position) might also need some synchronization between $\$ S 1$ 's events with $\$ S 2$ 's events

(inter-synchronization). The way to achieve this depends on the authoring tool. If the authoring tool does really manage event synchronization (which is rarely implemented), then this inter-synchronization can be automatically guaranteed. Otherwise, it should be guaranteed by an external agent, perhaps a person under her own responsibility.

\section{Rule 2.2: Color combinations}

Provided that both $\$ \mathrm{~S} 1$ and $\$ \mathrm{~S} 2$ have all their colors properly combined (i.e. there exists a high contrast between foreground and background colors in all elements within both $\$$ S1 and $\$$ S2), this could, however be broken if a bad color combination between a color from $\$ \mathrm{~S} 1$ and a color from $\$ \mathrm{~S} 2$ would be applied to any element of $\operatorname{comp}(\$ \mathrm{~S} 1, \$ \mathrm{~S} 2, \$$ position $){ }^{7}$ Figure 2 expresses the condition that all \$S2's elements having a foreground color but not a background color (or viceversa) will have a good color combination with the corresponding color inherited from $\$ \mathrm{~S} 1$. This formalized condition is only concerned on color combination derived from HTML color specification. ${ }^{8}$

\section{Rule 3.2: Grammar validation}

Most of the rules that should be followed in order to build accessible Web pages from accessible already built HTML

\footnotetext{
${ }^{7}$ This is only possible for elements within $\$ S 2$ that have provided a foreground color but no background color (or vice-versa). In that case, the non-specified color will be inherited from $\$ \mathrm{~S} 1$, which will be combined with the specified color within $\$ S 2$, so we must make sure that such a color combination will look have an acceptable color contrast. ${ }^{8}$ CSS-based color combination is outside the scope of a rule written in XPath [3] or XQuery [4], and it is also a difficultto-deal-with issue, since many color combination failures will reside on user's and author's external styles or default browser's configurations, not in Web documents themselves. As a result, CSS-based good color combination is outside the scope of Web composition, and must be guaranteed by a CSS-aware combination process.
} 
let $\$$ bgcolor1 := $\operatorname{pos}(\$ \mathrm{~S} 1, \$$ position $) / /$ ancestor-orself::*[@bgcolor][last()]/@bgcolor

return

let $\$$ fgcolor $1:=\operatorname{pos}(\$ \mathrm{~S} 1, \$$ position $) / /$ ancestor-orself::*[@color][last()]/@color

return

let $\$$ elemsf := $\$ \mathrm{~S} 2 / /$ font $[@$ color and not(ancestor-orself::*[@bgcolor])]

return

let $\$$ elemsb := \$S2//font[@bgcolor and not(ancestor-orself::*[@color])]

return

let $\$$ contrastbg1fg2 :=

every \$elemf in \$elemsf satisfies

highcontrast(\$bgcolor1, \$elemf/@color)

return

let $\$$ contrastbg $2 \mathrm{fg} 1:=$

every $\$$ elemb in $\$$ elemsb satisfies

highcontrast (\$fgcolor1, \$elemb/@bcolor)

return

\$contrastbg1fg2 and \$contrastbg2fg1

Figure 2: XPath pre-condition for disabling undesirable HTML-based color combinations for text

snippets rely on grammar validation. These rules should be followed in order to build valid documents that validate against a well known language, i.e., a HTML or XHTML [6] grammar. Every one of the following rules is described in a DTD or XML Schema for XHTML and must be followed in the Web Composition process in order to get accessible results:

1. $\$ \mathrm{~S} 2$ should fit grammatically into $\operatorname{pos}(\$ \mathrm{~S} 1, \$$ position $)$ according to the DTD or XML Schema being used for the final document being built. This implies that $\$ S 2$ should be allowed to be placed as a direct descendant part within the $\$ \mathrm{~S} 1$ 's node just above pos $(\$ \mathrm{~S} 1, \$$ position $)$. If $\$ \mathrm{~S} 2$ has a single root element, this root element should be an allowed child within $\$ S 1$ 's node just above $\operatorname{pos}(\$ S 1, \$$ position $) .^{9}$

2. No focusable elements should be nested. Focusable elements are those that can receive user's focus interaction (via mouse or keyboard or any other input device), like links or form fields. Provided that neither \$S1 nor \$S2 have nested focusable elements, the problem arises if comp $(\$ \mathrm{~S} 1, \$ \mathrm{~S} 2, \$$ position) has a forbidden combination of nested focusable elements derived from the composition process (i.e., a \$S2's focusable element within a $\$ S 1$ 's focusable element). In order to guarantee that Web Composition will not yield such forbidden nested combination, we must make sure that $\$ \mathrm{~S} 2$ has no focusable element or (if it has), that $\operatorname{pos}(\$ \mathrm{~S} 1, \$$ position $)$ is not within the domain of a focus-

\footnotetext{
${ }^{9}$ For example, table rows can only be inserted within a table (nowhere else), list items can only be inserted within a list (prohibited elsewhere), ... As a general rule, HTML tags are divided into block-type and inline-type, thus requiring that any block content is inserted only inside elements which are allowed to have block-type children. Generic tags like div or span are recommended to be used as a generic type of block-type or inline-type pieces respectively.
}

able element, like a link or a label. Figure 3 expresses condition with a XPath expression.

$\$ \mathrm{~S} 2 / /(\mathrm{a} \mid$ area $\mid$ label $\mid$ input $\mid$ select $\mid$ textarea $\mid$ button $)$ $=()$ or

$\operatorname{pos}(\$ \mathrm{~S} 1, \$$ position $) / /$ ancestor::(a | area | label | input | select $\mid$ textarea $\mid$ button $)=()$

Figure 3: XPath pre-condition for disabling undesirable nesting of focusable elements in Web composition

3. Forms should also be unnested. Figure 4 expresses the XPath condition that guarantees that comp $(\$ \mathrm{~S} 1, \$ \mathrm{~S} 2, \$$ position) will not have nested forms, provided that no nested form can be found within $\$ S 1$ nor $\$$ S2.

$\$ \mathrm{~S} 2 / /$ form $=()$ or $\operatorname{pos}(\$ \mathrm{~S} 1, \$$ position $) / /$ ancestor::form $=$ ()

Figure 4: XPath pre-condition for disabling undesirable nesting of forms in Web composition

4. If XHTML Basic $[7,8]$ is the target format of our page, no nested tables should be allowed either. Other HTML or XHTML variants allow nested tables, but if the final page should be a valid XHTML Basic page, nested tables are forbidden. Provided that neither $\$ S 1$ nor $\$$ S2 have nested tables, this prohibition is guaranteed as long as $\$ \mathrm{~S} 2$ has no table inside or, if it has, that it is not going to be inserted within a $\$ S 1$ 's table's scope, as depicted in figure 5

$\$ \mathrm{~S} 2 / /$ table $=()$ or $\operatorname{pos}(\$ \mathrm{~S} 1, \$$ position $) / /$ ancestor::table $=$ ()

Figure 5: XPath pre-condition for disabling undesirable nesting of tables in Web composition

5. No common $i d$ attributes, should be allowed. Because $i d$ attributes should be unique within a document, $i d$ attributes should not be commonly used in both $\$ \mathrm{~S} 1$ and $\$$ S2. Thus, the intersection of all $\$ S 1$ 's id values and all $\$ S 2$ 's $i d$ values should be the empty set. ${ }^{10}$ Figure 6 expresses such condition with a XPath expression.

6. Previous rule also applies to name attributes whenever used as id (i.e. when applied to elements depicted in figure 7), something which, in fact, is a deprecated feature which is explicitly forbidden in the most recent versions of XHTML (in favour of the $i d$ attribute).

7. Name attribute has other usages, though, when applied to other tags. No common name attributes in param tags should appear within the same object. Even though two different objects could share parameters with the same name, parameters for a single object

\footnotetext{
$\overline{{ }^{10} \$ \mathrm{~S} 1 / / *[@ i d] / @ i d \cap \$ S 2} / / *[@ i d] / @ i d=\emptyset$
} 
$\$ \mathrm{~S} 1 / / *[@ \mathrm{id}] / @ \mathrm{id}$ intersect $\$ \mathrm{~S} 2 / / *[@ \mathrm{id}] / @ \mathrm{id}=()$

Figure 6: XPath pre-condition for disabling the appearance of two elements with a common id in Web composition

$\$ S 1 / /($ a $\mid$ meta $\mid$ object $|\operatorname{img}|$ applet $|\operatorname{map}|$ form $) / @$ name intersect

$\$ \mathrm{~S} 2 / /(\mathrm{a} \mid$ meta $\mid$ object $|\operatorname{img}|$ applet $|\operatorname{map}|$ form $) / @$ name $=()$

Figure 7: XPath pre-condition for disabling the appearance of two elements with a common name in Web composition

should have different names, in order to be properly parametrized. Figure 8 illustrates that $\$ S 2$ 's orphan params inserted within a $\$ S 1$ 's object should not find a sibling param with a common name. Otherwise, two different params with a common name would be inside the same object. In other words, a document can have several param elements sharing a common name, but they should not be inside the same multimedia objects.

$\$ \mathrm{~S} 2 / /$ param[not (../(object|applet))]/@name intersect $\operatorname{pos}(\$ \mathrm{~S} 1, \$$ position $) /. . /($ object $\mid$ applet $) / /$ param/@name = ()

Figure 8: XPath pre-condition for disabling the common-named parameters for objects

8. Finally, no common name attributes in form fields (in those that must have unique names) should appear within the same form, as expressed in figure 9 This does not apply for radio or check-box buttons, however, because they can share a common name with other similar buttons.

\section{Rule 4.1: Language shifts}

Idiom changes within a document should be explicit. Provided that both $\$ S 1$ and $\$ S 2$ obey this rule, we still must guarantee that, if $\$ S 1$ 's default language is different from \$S2's default language, the xml:lang is used explicitly on the top of $\$ S 2$, as depicted in figure 10 .

Idiom changes between documents should also be explicit, i.e., the hreflang attribute should also be properly used. Though, assuming that both $\$ S 1$ 's and $\$ S 2$ 's links follow this rule, in order to guarantee that $\operatorname{comp}(\$ \mathrm{~S} 1, \$ \mathrm{~S} 2, \$$ position $)$ will also follow it, we still must make sure that all links within $\$ \mathrm{~S} 1$ or $\$ \mathrm{~S} 2$ (i.e., any compoundable piece of HTML page), properly use a hreflang if needed. This is a difficult issue for those authoring tools that have no means for specifying which idiom is being used, specially for those extracting data from heterogeneous and external information sources. In order to properly manage the hreflang attribute for every link, authoring tools should take into account the idiom of every target and the idiom of every link.
$\$$ S2//(textarea | select | button | input[@type != "radio" and @type!="checkbox"])[not (ancestor::form)]/@name intersect

$\operatorname{pos}(\$ \mathrm{~S} 1, \$$ position $) /$ ancestor-or-self::form[last ()$] / /($

textarea | select | button | input[@type != "radio" and @type!="checkbox"])/@name

$=()$

Figure 9: XPath pre-condition for disabling the common-named form fields

\section{\$S2/@xml:lang or}

pos $(\$ \mathrm{~S} 1, \$$ position $) / /$ ancestor-or-

self::*[@xml:lang][last()]/@xml:lang = lang $(\$ S 2)$

Figure 10: XPath pre-condition for requiring $\mathrm{xml}$ lang attributes (language shifts)

\section{Rule 4.2: Proper abbreviations and acronyms}

Abbreviations and acronyms should be properly used. This requires, among other things, that any single text defined with an abbreviation or acronym is specified only once at most within a document. ${ }^{11}$ In other words, $\$$ S1 and $\$$ S2 should have no common abbreviation or acronym's text within a document, as depicted from figure $11 .^{12}$

$(\$ \mathrm{~S} 1 / /$ abbr/text () union $\$ \mathrm{~S} 1 / /$ acronym/text ()$)$ intersect

$(\$ \mathrm{~S} 2 / /$ abbr/text () union $\$ \mathrm{~S} 2 / /$ acronym/text ()$)$ $=()$

Figure 11: XPath pre-condition for avoiding undesirable repeated abbreviations or acronyms

\section{Rule 9.4: Unique and consecutive tabulation order}

Tabulation order should be consistent, if specified. This requires that no more than a single element is focusable for a given tabulation order. As a result, $\$ \mathrm{~S} 1$ and $\$ \mathrm{~S} 2$ should have no common tabindex attribute, as depicted in figure $12 .^{13}$

\section{Rule 9.5: Unique shortcuts}

Shortcuts should also be unique, as expressed in figure $13 .{ }^{14}$

\section{Rule 10.5: Non consecutive links}

Provided that all $\$$ S1's and $\$ S 2$ 's links have no consecutive links (some printable text between links), their composition could have consecutive links without such printable characters if a $\$$ S2's link appears just in front of a $\$ S 1$ 's link. For this rule, XPath is not enough, but a combination of XPath and XPointer [5] operators can formalize this condition, as

\footnotetext{
${ }^{11}(\$ S 1 / /$ abbr/text ()$\cup \quad \cup S 1 / /$ acronym $/ \operatorname{text}())$ $(\$ \mathrm{~S} 2 / / \mathrm{abbr} / \operatorname{text}() \cup \$ \mathrm{~S} 2 / /$ acronym $/ \operatorname{text}())=\emptyset$
}

${ }^{12}$ In fact, this should be applied not only to a page, but to the related set of pages.

${ }^{13} \$ \mathrm{~S} 1 / / * / @$ tabindex $\cap \$ \mathrm{~S} 2 / / * /$ @tabindex $=\emptyset$

${ }^{14} \$ \mathrm{~S} 1 / / * / @$ accesskey $\cap \$ \mathrm{~S} 2 / / * / @$ accesskey $=\emptyset$ 
$\$ \mathrm{~S} 1 / / * / @$ tabindex intersect $\$ \mathrm{~S} 2 / / * / @$ tabindex $=()$

Figure 12: XPath pre-condition for avoiding elements with a common tabulation order

$\$ \mathrm{~S} 1 / /^{*} / @$ accesskey intersect $\$ \mathrm{~S} 2 / /^{*} / @$ accesskey $=()$

Figure 13: XPath pre-condition for avoiding elements with a common shortcut

depicted in figure $14 .^{15}$

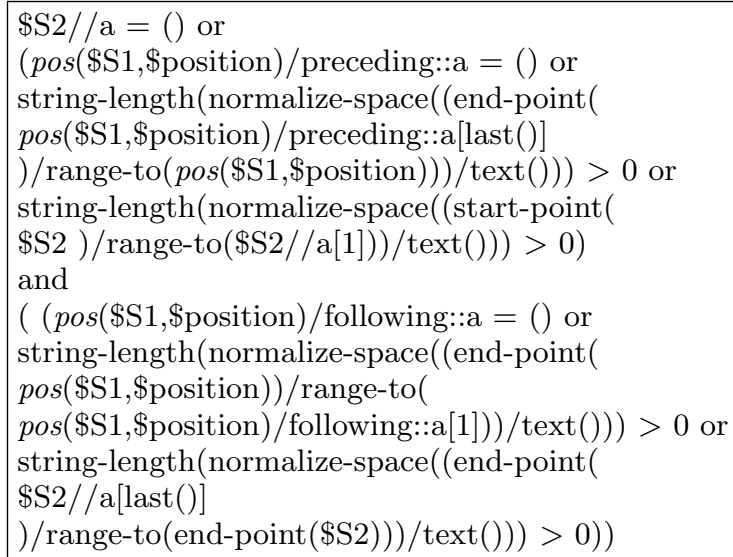

Figure 14: XPath + XPointer pre-condition for avoiding consecutive links without printable nonlinkable characters between them

\section{Rule 13.1: Clear links}

There should be no links sharing both a text and a title but pointing to different targets. ${ }^{16}$ Provided $\$$ S1 and $\$$ S2 have no such ambiguous links, there exist a functional dependency such that for every pair of (link's contents, link's title) only a single target may be found in both \$S1 and \$S2. In that case, we should also make sure that no link in $\$ \mathrm{~S} 1$ is similarly described in $\$ S 2$ (and pointing to a different target), or vice-versa. If so, an ambiguity would be introduced in the composed result. Figure 15 describes such condition.

\section{Guaranteed-by-process checkpoints}

Previous pre-conditions should be met in order to guarantee propagation of WAI compliance from $\$ S 1$ and $\$ S 2$ to the composition result of both pieces. Those rules are required to be followed for this propagation. On the other hand, any other checkpoint not depicted in section 3 will be automatically propagated. In other words, if both \$S1 and $\$ S 2$ follow these checkpoints, comp $(\$ S 1, \$ S 2, \$$ position $)$ will surely follow them too. Here are some examples and their explanations. We don't include all of them. They are all WCAG which are not included within section 3 .

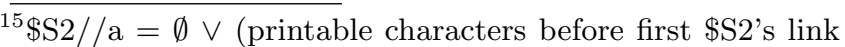
$\wedge$ printable characters after last $\$$ S2's link)

${ }^{16}$ This would lead to ambiguity, because two similar starting roads point to different targets.
}

(every $\$$ a1 in $\$ \mathrm{~S} 1 / /$ a satisfies

$\$ \mathrm{~S} 2 / / \mathrm{a}[\operatorname{text}()=\$ \mathrm{a} 1 / \operatorname{text}()$ and @title $=\$ \mathrm{a} 1 / \mathrm{Qtitle}$ and @href !=\$a1/@href] = ()) and

(every $\$ a 2$ in $\$ S 2 / /$ a satisfies

$\$ \mathrm{~S} 1 / / \mathrm{a}[\operatorname{text}()=\$ \mathrm{a} 2 / \operatorname{text}()$ and @title $=\$ \mathrm{a} 2 /$ @title and @href $!=\$ \mathrm{a} 2 / @$ href] $=())$

Figure 15: XPath pre-condition for avoiding ambiguous links

\section{Rule 1.5: Redundant links for client image maps}

Provided that both $\$ S 1$ and $\$ S 2$ have redundant links for their client image maps (areas), the composition result of $\$ S 1$ and $\$ S 2$ will also have those redundant links (at least), thus it will also comply WCAG 1.5.

PROVIDED that snippets have redundant links for their areas, i.e.

$\$ S 1 /$ area[let $\$$ href $:=$ self::area/@href return

$\operatorname{count}(\$ \mathrm{~S} 1 / / \mathrm{a}[@$ href $=\$ \mathrm{href}])=0]=() \wedge$

$\$ \mathrm{~S} 2 /$ /area[let $\$$ href $:=$ self::area/@href return $\operatorname{count}(\$ \mathrm{~S} 2 / / \mathrm{a}[@$ href $=\$$ href $])=0]=()$

WE CONCLUDE that composition result will also have redundant links comp $(\$ S 1, \$ S 2, \$$ position $) / /$ area[ let \$href:=self::area/@href return $\operatorname{count}(\operatorname{comp}(\$ \mathrm{~S} 1, \$ \mathrm{~S} 2, \$$ position $) / / \mathrm{a}[@ \mathrm{href}=\$ \mathrm{href}])=0]=()$

BECAUSE those links will still be there $\operatorname{comp}(\$ \mathrm{~S} 1, \$ \mathrm{~S} 2, \$$ position $) / / \mathrm{a}=\$ \mathrm{~S} 1 / / \mathrm{a} \cup \$ \mathrm{~S} 2 / / \mathrm{a}$

\section{Rule 12.4: Labels}

Provided that both $\$ \mathrm{~S} 1$ and $\$ \mathrm{~S} 2$ have no label shared by two form fields, the composition result of $\$ \mathrm{~S} 1$ and $\$ \mathrm{~S} 2$ will not have any label shared by two form fields, because it is not possible that the other piece brings a form field whose id attribute is equal to any other element already used. So, because id attributes are required to be unique (see rule 3.2: validation), the number of form fields pointed by a label can not incidentally be increased in the composition process. It must be 1 . Otherwise a validation error message spotting that two elements share a common id will appear.

PROVIDED that snippets have proper labels, i.e. $\$$ S1//label[let $\$ 1:=$ self::label return $\operatorname{count}(\$ \mathrm{~S} 1 / /($ select $\mid$ input $\mid$ textarea $)[@ i d=\$ 1 / @$ for $]) !=1]$ $=() \wedge$

$\$ \mathrm{~S} 2 / /$ label[let $\$ 1:=$ self::label return $\operatorname{count}(\$ \mathrm{~S} 2 / /($ select $\mid$ input | textarea $)[@ i d=\$ 1 / @$ for $]) !=1]$ $=()$

WE CONCLUDE that composition result will also have proper labels $\operatorname{comp}(\$ \mathrm{~S} 1, \$ \mathrm{~S} 2, \$$ position $) / /$ label[let $\$ 1$ =self::label return $\operatorname{count}((\operatorname{comp}(\$ \mathrm{~S} 1, \$ \mathrm{~S} 2, \$$ position $) / /$ (select | input | textarea) $[@ i d=\$ 1 / @ f o r]) !=1]=()$

BECAUSE $i d$ attributes must be unique

\section{Rule 12.4: Form fields}

Provided that both $\$ \mathrm{~S} 1$ and $\$ \mathrm{~S} 2$ have no form field shared by two labels, the composition result of $\$ \mathrm{~S} 1$ and $\$ \mathrm{~S} 2$ will have 
no form fields shared by two labels, because it is not possible that the other snippet brings a label whose for attribute is equal to any other element already used. Only if such label was not associated to any form field, we could have a valid document that presents a form field having more that one associated label. However, this is not possible provided the conditions from previous rule 12.4 for labels.

PROVIDED that snippets have proper form fields, i.e. $\$ \mathrm{~S} 1 / /$ (select | textarea | input[@type="text" or

@type="password" or @type="radio" or

@type="checkbox"])[let $\$$ ff:=self::node()

return

$\operatorname{count}(\$ S 1 / /$ label[@for=\$ff/@id] $) !=1]=() \wedge$

$\$ \mathrm{~S} 2 / /$ (select | textarea | input[@type="text" or

@type="password" or @type="radio" or

@type="checkbox"])[let $\$ \mathrm{ff}:=$ self::node()

return

$\operatorname{count}(\$ \mathrm{~S} 2 / /$ label $[@$ for $=\$ \mathrm{ff} / @ \mathrm{id}]) !=1]=()$

WE CONCLUDE that composition result will also have proper snippets

$\operatorname{comp}(\$ \mathrm{~S} 1, \$ \mathrm{~S} 2, \$$ position $) / /($ select $\mid$ textarea |

input[@type="text" or @type="password" or

@type="radio" or @type="checkbox"])[let \$ff:=self::node() return

$\operatorname{count}(\operatorname{comp}(\$ \mathrm{~S} 1, \$ \mathrm{~S} 2, \$$ position$) / /$ label[ $@$ for $=\$ \mathrm{ff} / @ \mathrm{id}]) !=1]$ $=()$

BECAUSE $i d$ attributes must be unique

\section{Other checkpoints}

All other checkpoints not previously mentioned, though not detailed fall within this category. The existence of alternative contents and the proper usage of headings, lists or cites are characteristics that will never be broken by the Web Composition process. ${ }^{17}$

\section{WCAG support}

XPath and XQuery expressions from previous sections spot HTML nodes and attributes having accessibility problems. These elements should be properly managed by an authoring tool, so that author's attention can be directly brought to these barriers in a semi-automated edition process like our WSLS platform, making easier the creation of accessible contents.

\subsection{Aspects of a Supporting System}

The high availability of processors and tools to apply the presented XML-based technologies eases the implementation of the formalized rules. Unfortunately, , not all WCAG rules can be expressed with W3C standards like XPath or XPointer. Sections 3 and 4 introduced a set of checkpoints that exceeds the capabilities of such functional query languages. To automate or at least semi-automate, these checkpoints demand for a supporting system that facilitates compliance in the integration of Web contents obtained from distributed heterogeneous sources by process and by architecture. Compliance by process means that checkpoints are controlled by a system providing dedicated processes. On the other hand, compliance by architecture relies on the

${ }^{17}$ As long as snippets are accessible and XHTML validation is achieved. overall structure of the system and its design decisions to keep checkpoints or provide mechanisms to formulate them. Formalizing the checkpoints from sections 3 and 4 requires for imperative programming languages like Java, C\# or $\mathrm{C}++$ to express the complex algorithms. Furthermore, the supporting system must provide additional environment information. This information can be of different fashion concerning the audience, the user agent or even constraints which a user is operating under, e.g. noisy surrounding or hands-free environment.

\subsection{Web-Composition Service Linking System}

The Web-Composition Service Linking System (WSLS) [14] is a component-based system which applies the service-oriented paradigm. It supports issues of composing, discovering and reusing services. A WSLS-based service is a maintainable, manageable and configurable building block. Hence, it forms a unit for reuse and allows the composition of Web applications similar to the building block principle. The process of composing such services is guided in a systematic way by the underlying WSLS framework. Figure 18 depicts the general form of each service within WSLS. A service is understood as a component that consists of a set of service elements. A service element achieves dedicated tasks corresponding to its classification. The classification scheme provides six distinguished categories: Data, Presentation, Navigation, User Interaction, Process and Communication. The six elements are mediated by a service control function. The WSLS approach follows the separation of concerns principle.

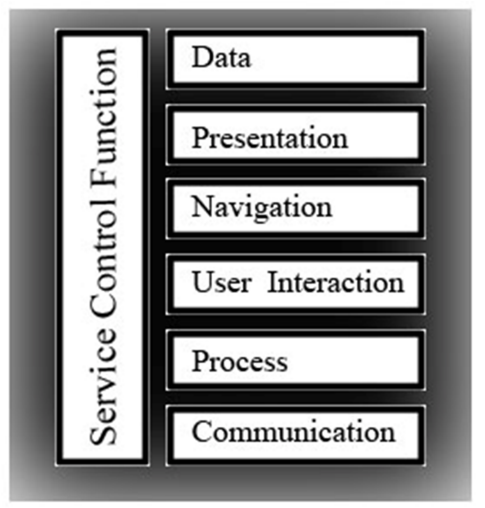

Figure 16: WSLS follows the separation of concerns principle to decompose complexity and control accessibility

Beyond the advantage of the reuse aspect of these components, the separation of concerns facilitates also being compliant to underlying guidelines. Thus, the presentation of a service can be adapted in a fast and efficient fashion to local prevailing presentation constraints. Furthermore, besides presentation guidelines that simply concern the layout of services, more powerful rules regarding the accessibility of a service can be realized, like e.g. U.S. Section 508 Guidelines [13].

Providing services through the Web is rapidly becoming the emerging trend. As more and more governments adopt laws regarding accessibility, enterprises are also affected. While some laws, like Section 508, currently impact only the US. federal government, there is even a trickle-down effect 
to private enterprises. Companies with contracts to supply government with products stand to lose their business if the products are not accessible or suitable for creating accessible Web sites. WSLS faces these requirements by providing support for designing and implementing accessible building blocks as well as procedures for their composition.

\subsection{WCAG support in WSLS}

The WSLS framework is implemented on top of the .NET framework. Therefore, the support of XML technologies like XPath or XSL(T) is given and can be applied systematically. Hence, we started implementing the automatable checkpoints regarding these technologies. The realization of the unaffected rules stated in sections 3 and 4 poses a non-trivial challenge at the underlying supporting system. Subsequently, we show for some selected rules how WSLS supports the abidance on these.

\section{Rule 13.2: "Provide meta-data"}

"Providing additional data to add semantic information to pages and sites."

Figure 19 depicts the general structure of a data element as prescribed in the WSLS framework. Specialized data objects can be created by inheriting from the base DataObject class to fulfill dedicated tasks. By default, each data element used in WSLS possesses meta-data. This comprises at least the Dublin Core standard [18]. In order to support other specifications needed to satisfy arbitrary environments, these objects are also extensible. Thus, each data object can provide specialized meta-data, if necessary. This is particularly useful in different scenarios, e.g. to indicate a document's author, content type together with the last modification date. Furthermore, this extensible approach allows for enhanced scenarios where the semantic description is reused by other processes.



Figure 17: Prescribed structure of a data element in WSLS

\section{Rule 4.1: "Identify changes in the natural language"}

"Clearly identify changes in the natural language of a document's text and any text equivalents (e.g., captions)."

As described above, each data element provides at least Dublin Core meta-data. This information is used to provide language relevant information for presented data. Figure 20 depicts a Web application that was built on top of the WSLS framework. The scenario shows a news service that displays recent news items. WSLS provides standard user interaction behaviour like creating, editing and deleting data elements of a given service. Therefore, the system even supports the automatic generation of forms for editing data elements of standardized Web services. The process of editing such a data element is demonstrated.

Besides the application of the presented formalization rules, the content author is prevented from writing wrong or insufficient specified HTML code by supporting guiding processes that query for the necessary data. This data is automatically incorporated into the HTML output as prescribed by the corresponding formalization rule. In this example the author is asked for the specific language of the content provided.

\section{Conclusions and future work}

This article has presented conditions formalized with W3C standards that guarantee that the Web Composition [12] approach will always yield accessible contents if accessible ingredients are provided. As expressed, there are some accessibility conditions that will be inherited from smaller pieces and Web Composition guarantees by process (section 4), but other accessibility features require extra conditions in order to be kept. We have formalized the conditions that will avoid that accessibility barriers might be introduced by the Web Composition process itself. We introduced WSLS, a supporting system that affords compliance to these rules by process and by architecture. The realization of selected rules in WSLS yielded already to promising results. For the future we plan to adopt architectural issues as well as augmented processes that guide the design, implementation and evolution of sites and applications towards the vision of the compliant and accessible Web.

\section{Acknowledgements}

The work reported in this paper has been partially funded by the projects INFOFLEX TIC2003-07208 and SIEMPRE TIC2002-03635 of the Spanish Ministry of Science and Research.

\section{REFERENCES}

[1] W3C Web Content Accessibility Guidelines 1.0 www . w3. org/TR/WCAG10

[2] W3C Techniques For Accessibility Evaluation And Repair Tools W3C Working Draft, 26 April 2000 www.w3.org/TR/AERT

[3] W3C XML Path Language (XPath) Version 1.0 W3C Recommendation 16 November 1999 www.w3.org/TR/xpath

[4] W3C XQuery 1.0: An XML Query Language W3C Working Draft 29 October 2004 www.w3.org/TR/xquery

[5] W3C XML Pointer Language (XPointer), W3C Working Draft 16 August 2002 www . w3. org/TR/xptr

[6] W3C XHTML 1.0 TM The Extensible HyperText Markup Language (Second Edition), A Reformulation of HTML 4 in XML 1.0, W3C Recommendation 26 January 2000, revised 1 August 2002 www . w3. org/TR/xhtml1

[7] W3C XHTML Basic W3C Recommendation 19 December 2000 www.w3.org/TR/xhtml-basic 


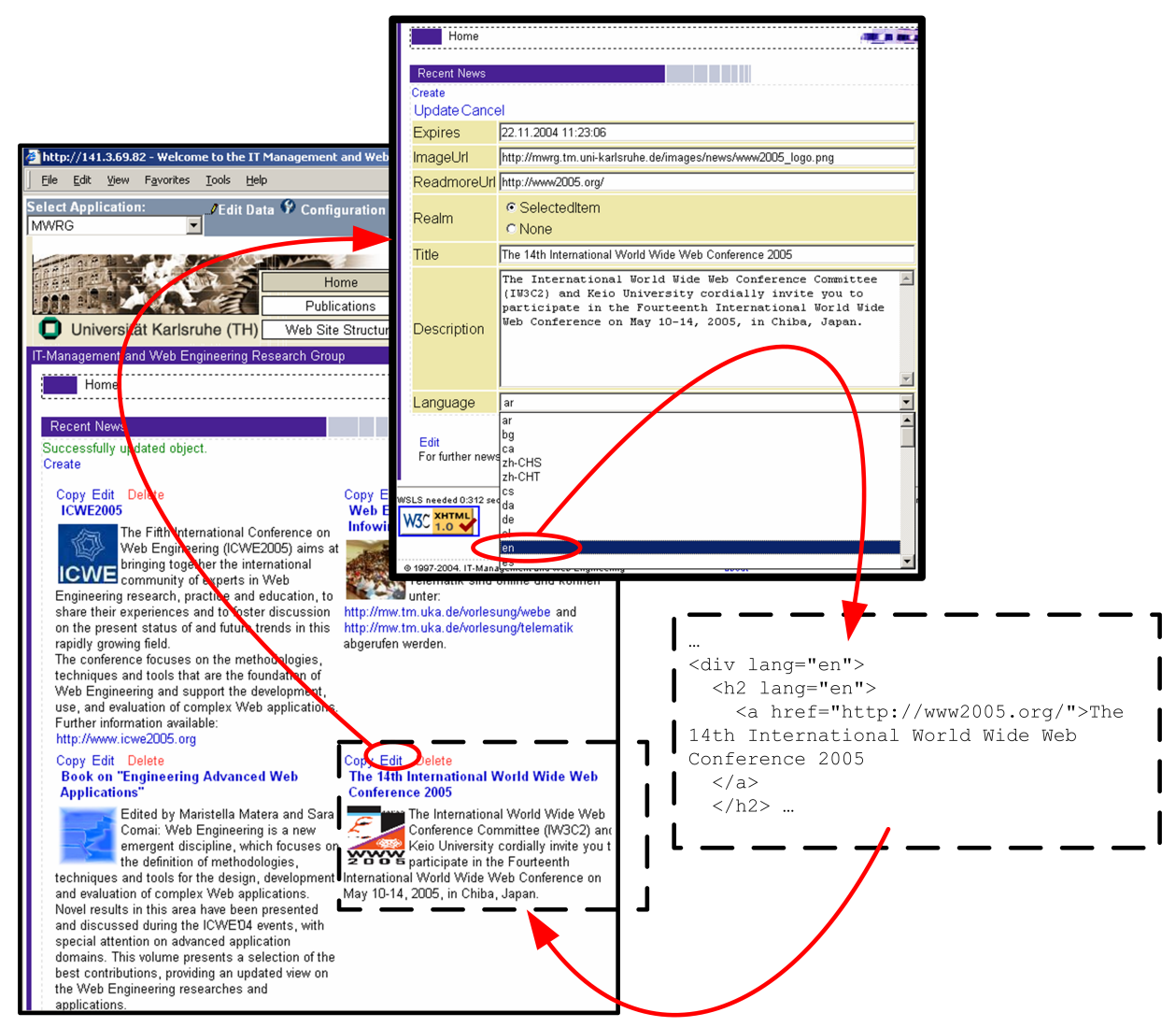

Figure 18: Using metadata to provide natural language relevant information

[8] W3C Markup Validation Service validator.w3.org

[9] Watchfire Bobby Accessibility tool bobby. watchfire.com/bobby/html/en/index.jsp

[10] CEAPAT, Fundación CTIC, Spanish Ministry of Employment and Social Affairs (IMSERSO) Online Web accessibility test www. tawdis.net

[11] Fondazione Ugo Bordoni Torquemada, Web for all www . webxtutti.it/testa_en.htm

[12] Hans-Werner Gellersen, Robert Wicke and Martin Gaedke WebComposition: An object-oriented support system for the Web engineering lifecycle

Computer Networks and ISDN Systems, Vol 29, 8-13, 1997, pages 1429-1437

[13] Center for IT Accommodation (CITA) U.S. Section 508 Guidelines www. section508.gov

[14] Gaedke, M., Nussbaumer, M., and Meinecke, J. WSLS: An Agile System Facilitating the Production of Service-Oriented Web Applications

Engineering Advanced Web Applications, M. Matera and S.

Comai, Editors. 2004, Rinton Press 